National and Trust IPC standards. Following reported cases of MRSA bacteraemia and colonisation, and MRSA cases missed through previous routine screening regimes in our Trust, the Team has implemented mandatory routine MRSA screening for patients who fulfilled locally-defined high-risk criteria. This audit aims to determine the standard of MRSA screening for these high-risk patients on the Neonatal and Acute Paediatric inpatient wards.

Methods Patients admitted to Bluebell (Acute Paediatric) ward, the Children's Assessment Unit (CAU) or the Neonatal unit (NNU) were retrospectively identified and compared against a locally-defined set of criteria for patients at high risk of MRSA colonisation or infection. The criteria included previous MRSA infection or colonisation, transfer from or previous admission in another hospital, recurrent skin lesions or infections, and exposure to family member with known MRSA. The study began in May 2019 with data collected up to midOctober 2019. Electronic pathology records were then searched. The assumed target of MRSA testing, where appropriate, was $100 \%$, with the Trust's IPC standard of $>90 \%$.

Results Preliminary results yielded 136 high-risk patients and 145 appropriate instances for screening. An overall screening rate of $78.6 \%(114 / 145)$ was noted covering all areas during the audit period. The Neonatal Unit had the highest rate of screening at $97.6 \%(41 / 42)$, followed by CAU at $80 \%(16 / 20)$ and Bluebell at $68.7 \%$ (57/83). Month-by-month analysis showed June to have the lowest rate for all areas $(50.0 \% 8 /$ 16) and August with the highest rate (95.0\% 19/20).

Conclusion The audit highlighted that compliance with guidelines is variable across areas and different months. Despite our study not being able to consider the many factors that contribute towards this, the data has identified gaps in current practice and will guide the goals on education and training of clinical staff regarding IPC guidelines, with the focus on MRSA high risk screening criteria, to ensure robust and safe practice.

\section{G135(P) NATIONAL IMPACT OF RCPCH BEST PRACTICE GUIDE ON TIMING OF DETECTION OF CLEFT PALATE - TIME TO ADOPT SUCCESSFUL LOCAL QUALITY IMPROVEMENT INITIATIVES}

${ }^{1} \mathrm{H}$ McElroy, ${ }^{2} \mathrm{C}$ Marsh, ${ }^{3} \mathrm{R}$ Bailey, ${ }^{3} \mathrm{~J}$ Leigh, ${ }^{4} \mathrm{~S}$ Deacon, ${ }^{4} \mathrm{~J}$ Medina. ${ }^{1}$ Medway NHS Foundation Trust; ${ }^{2}$ South West Cleft Service; ${ }^{3}$ South Thames Cleft Service, GSTT, 4 CRANE Database

\subsection{6/archdischild-2020-rcpch. 108}

Aim Historical data show that $30 \%$ of cleft palates (CP) are not identified within $24 \mathrm{hrs}$ of birth, the national standard. In 2014 the RCPCH launched a Best Practice Guide (BPG) recommending a change to examination of the palate from digital palpation to visual inspection to improve detection rates. ${ }^{1}$ We report the national impact of the BPG, highlighting regional variations, and share solutions from a local quality improvement programme (QIP).

Method Data on diagnosis time of CP for 2013-2018 were identified from CRANE database ${ }^{2}$ (England, Wales, Northern Ireland) and supplemented with data from Scotland and Republic of Ireland to explore regional variations. Local data from Medway Hospital, Kent, 2010-2019 were collated for a Quality Improvement Project to improve early detection of cleft palate.
Results Nationally there has been a significant increase in detection CP $<24$ hrs age since the BPG: 2013-14 births (67\%) v. $2015-16$ births (71\%) v. $2017-18$ births $(75-76 \%)$ [p <0.001]. Locally Medway Hospital: 30\% CP (4/13) were diagnosed within 24 hrs of birth between 2010-2014. Since introducing the QIP all cleft palates $(n=11)$ from 2015-2019 have been identified within 24 hrs of birth. ${ }^{3}$

Conclusion The timing of detection of cleft palate has improved since publication of the RCPCH Best Practice Guide. Late diagnosis remains an issue for quality of care and family experience. Local QIP can successfully sustain local detection rates at the national standard. We advocate adoption and spread of quality improvement initiatives to complement the BPG.

\section{REFERENCES}

1. RCPCH. (2014). Palate examination: Identification of cleft palate in the newborn Available at https://www.rcpch.ac.uk/resources/palate-examination-identificationcleft-palatenewborn-best-practice-guide (Accessed: 7 July 2020).

2. Royal College Surgeons. (2020). CRANE Database 2019 Annual Report. Available at https://crane-database.org.uk/ (Accessed: 7 July 2020).

3. McElroy $\mathrm{H}$, Habel $\mathrm{A}$, Jokinen $\mathrm{M}$, et al. Improving the early detection of cleft palate in the UK. Infant 2017;13:223-27.

\section{G136(P) JUNIOR-LED IMPROVEMENT PROJECT: IMPROVING MORNING HANDOVERS QUALITY AND LENGTHS IN A DISTRICT GENERAL HOSPITAL WITH THE INTRODUCTION OF A HANDOVER CHECKLIST}

S Tran, A Davies. Children's Services (Paediatrics), West Middlesex University Hospital, Chelsea and Westminster Hospital NHS FT, London, UK

\subsection{6/archdischild-2020-rcpch.109}

$\mathrm{RCPCH}$ worked nationally on a Situation Awareness for Everyone (S.A.F.E) toolkit that promoted safer handovers for patient safety. Due to the number of clinical areas covered and personal preferences, departmental handovers always varied in duration, structure and consistency. Key questions regarding staffing levels or unwell children would only be mentioned when issues arose.

Aim This project aimed to improve situation awareness for staff and to improve patient safety through structuring handovers to be more efficient.

Method The major invention was introducing a handover checklist to signal the start of handover covering many important aspects: staffing issues, potential risks to clinical resources - i.e. high-risk deliveries, HDU-level patients, busy emergency department and bed numbers. Start and end times were also documented.

Many iterations utilising improvement science occurred, including varying what was covered on the checklist, who fills it out and seeking departmental approval to separate midweek handovers for the neonatal and paediatric teams.

The latest checklist consists of: start/end times, staffing levels, 'Sickies'/HDU patients, any outgoing/pending transfers, high-risk deliveries, paediatric ED status, bed status, new safeguarding concerns and whether split handovers should occur.

Results The project is still running but over the first 20-week period, the checklist was filled in $80 \%$ of the time. Only $50 \%$ (41/81 documented) of handovers started at $8.30 \mathrm{am}$. Monday handovers took on average 65 minutes compared to 30-35 minutes on other days. Staffing issues were highlighted 
in $56 \%$ of handovers - including rota gaps, sickness and cancelled locums.

The non-clinical rota coordinator now feels empowered to raise key staffing issues and the staff have commented that it has provided a good snapshot of all the clinical areas. Data from the next 20-week period and a further qualitative survey on how it has improved staff's situation awareness are being compiled.

Conclusion The introduction of a handover checklist can provide a structure and give staff an overview of the department's clinical risk, therefore it can improve patient safety. Whilst it is easy to obtain numerical data on the length of handovers and whether key issues were discussed, further work is required to delineate secondary outcomes - staff's situational awareness/bandwidth and impact on departmental teaching.

\section{G137(P) FOOD MATTERS: A MULTIDISCIPLINARY INITIATIVE TO RAISE FOOD ALLERGY AWARENESS AND SAFETY ON A PAEDIATRIC WARD}

I Gerry, A Freedman, S Green, K Gudka, L Gibb, H Dejesus, E Lee, M Gandhi. Paediatrics, Royal Free Hospital, London, UK

\subsection{6/archdischild-2020-rcpch.110}

Background Increasing numbers of children who attend hospital have co-existing food allergies, whilst the incidence of children with food allergies worldwide continues to rise. Anxiety and confusion exist amongst these patients, carers and healthcare staff about food allergy awareness. There is currently no national universal training programme in place for healthcare professionals and other staff members.

Objectives

- To improve the safety of children with food allergies attending the paediatric ward

- To develop a structured training tool and implement a universal training programme for all healthcare professionals and staff.

Methods We undertook a quality improvement project to develop a structured teaching tool to train ward staff at all levels on food allergens and labelling, identification of children with food allergies and acute allergy management. A multidisciplinary team involving doctors, nurses, dietician, housekeepers, teachers and physician assistants worked on this initiative. Teaching was facilitated using microteach face to face sessions to ensure maximum staff uptake on the 'shop floor'. A pre and post-teaching questionnaire was undertaken to evaluate the efficacy of the teaching sessions.

Results 23 members of staff were trained including nurses, doctors, housekeepers, ward teachers, caterers and dieticians. During training, we identified that 22/23 (95\%) of staff members were willing to guess what food allergens were in a processed food based on prior knowledge without checking the label. Prior to training 9/23 staff members had difficulty understanding food allergen labelling and laws. We were able to address common misconceptions during training using practical examples. During training staff were taught to identify symptoms of food allergy and how to use an adrenaline autoinjector. After training 12/23 (52\%) said that they felt much more knowledgeable about food allergies. 22/23 (95\%) staff members trained felt confident in when and how to use an adrenaline autoinjector compared to $13 / 23$ prior to training.

Conclusions Implementing a universal food allergy awareness programme for all staff on the ward where food is eaten and enjoyed is an important step towards safeguarding children on the ward with food allergies.

\section{G138(P) UTILISING SIMPLE TECHNOLOGY TO IMPROVE MANAGEMENT OF TYPE 1 DIABETES PATIENTS}

${ }^{1} \mathrm{D}$ Dodia, ${ }^{2} \mathrm{~S}$ Wassouf, ${ }^{2} \mathrm{M}$ Watson. 'Medicine, Imperial College London, London, UK; ${ }^{2}$ Paediatric Diabetes, St. Mary's Hospital, London, UK

\subsection{6/archdischild-2020-rcpch.111}

Background and Aims The Paediatric Diabetes team of an inner-city hospital use Microsoft Excel to record Haemoglobin A1c (HbA1c) measurements from 116 patients. HbA1c levels inform the clinician of long-term glycaemic control and allow adaptation of the patient management plan.

Manual editing spreadsheets is an arduous process with a risk of human error. IT literacy of staff, training times, and costs limit the use of technological advances. Microsoft Excel - a cheap, simple, and easily accessible program - empowers teams to create dynamic spreadsheets. We aimed to increase the time efficiency of the Paediatric Diabetes team by improving their spreadsheet.

Methods The original HbA1c spreadsheet was assessed using a questionnaire and team discussion. The spreadsheet was amended based on team requirements and suggestions. Data grouping was used to simplify the spreadsheet. Conditional formatting highlighted missing data and appointments, upcoming appointments, and colour coded $\mathrm{HbA} 1 \mathrm{c}$ values within certain ranges. Summaries were evaluated using 'COUNTIF' and 'AVERAGE' formulae. Four hours were spent by a medical student implementing these changes.

Results Feedback from the team was extremely positive, with one person commenting on the intuitiveness and easeof-use. Post-implementation questionnaire responses noted that the spreadsheet had higher accuracy and improved layout. $100 \%$ of participants reported the new spreadsheet was easier to use. The time taken for data input by healthcare professionals reduced by $77.7 \%$ (from 45 to 10 minutes per month).

Conclusions Better data presentation highlighted patients with higher HbA1c levels to healthcare professionals, therefore allowing further support to be provided and thus improving patient care. Automation of formatting and spreadsheet summarising reduced time spent on the document and enabled healthcare professionals to arrange appointments with ease. An explanation of any complex formulae used was provided, to ensure that the document remains usable to those without high IT proficiency.

This idea could be adapted to various teams, especially those managing chronic conditions. Clinicians could consider investing time in straightforward programs like these, as they can be effective at a lower cost than new technological advances.

\section{G139(P) ABSTRACT WITHDRAWN}

